\title{
Prolonged Survival of a Patient With Pelvic Recurrence of Ovarian Malignant Mixed Mullerian Tumor After Chemoradiotherapy
}

\author{
Fatemeh Homaei Shandiz ${ }^{1}$; Sima Kadkhodayan ${ }^{2,}$; Malihe Hsanzade Mofrad ${ }^{2}$;Zohre Yousefi \\ Roodsari ${ }^{2}$; Noorieh Sharifi Sistani ${ }^{1}$; Majid Nabizadeh Marvast ${ }^{3}$; Mahbobe Sadeghei ${ }^{1}$ \\ ${ }_{1}^{1}$ Research Center of Solid Tumor Treatment, Ghaem Hospital, Mashhad University of Medical Sciences, Mashhad, IR Iran \\ ${ }_{3}^{2}$ Research Center of Women's Health, Ghaem Hospital, Mashhad University of Medical Sciences, Mashhad, IR Iran \\ ${ }^{3}$ Mashhad University of Medical Sciences, Mashhad, IR Iran \\ ${ }^{*}$ Corresponding Author: Sima Kadkhodayan, Research Center of Women's Health, Ghaem Hospital, Mashhad University of Medical Sciences, Mashhad, IR Iran. Tel: +98-5118012477, \\ Fax:+98-5118430569, E-mail: kadkhodayans@mums.ac.ir
}

Received: September 18, 2013; Revised: April 27, 2014; Accepted: July 13, 2014

\begin{abstract}
Introduction: Malignant Mixed Mullerian Tumor (MMMT) is a very rare tumor, accounting for less than $1 \%$ of all ovarian cancers. Case Presentation: We present a 64-year-old woman with stage III MMMT of ovary that was treated with platinum-based chemotherapy after optimal cytoreductive surgery. After 25 months of being disease free, she had a pelvic recurrence and a good response to chemoradiotherapy.

Conclusions:Optimal cytoreductive surgery and chemotherapy maybe the best treatment in MMMT butmore discussion and experiences are needed regarding the effectiveness of radiotherapy.
\end{abstract}

Keywords:Mixed Tumor, Mullerian; Cytoreductive Surgery; Chemoradiotherapy; Chemotherapy; Ovary

\section{Introduction}

Malignant Mixed Mullerian Tumor (MMMT) of ovary is a rare tumor, accounting for less than $1 \%$ of all ovarian cancers, that most commonly occurs in postmenopausal women with low parity (1). It is a biphasic tumor, composed of both epithelial and stromal components (2). Predominant stromal tumor has been associated with worse outcome and poor response to treatment. Known risk factors for MMMT include obesity, nulliparity, exogenous estrogen, and long-term tamoxifen use (3). Stage of disease is the most important survival-determining factor in patients with MMMT (4). Complete surgical resection with staging is the main treatment of this tumor. Although there is no standard regimen for chemotherapy, it is advised in patients with stage II or higher (1). The role of adjuvant radiotherapy is controversial (5). In this report, we introduce a case of prolonged survival of a patient with pelvic recurrence of ovarian MMMT after chemoradiotherapy.

\section{Case Presentation}

In January 2007, we admitted a 64-year-old woman with acute abdominal pain, nausea and vomiting in Ghaem Hospital, Mashhad University of Medical Sciences, Iran. She had low appetite and inability in passing stool and gas in the recent week. On physical examination, she had a distended abdomen and on abdominal radiography, there were multiple air-fluid levels, suspected of bowel obstruction. At laparotomy with a large midline incision, there was adhesion of omentum and bowels on a large heterogeneous pelvic mass. After releasing the adhesions, right ovarian tumor was revealed. Cytoreductive surgery by transabdominal hysterectomy and bilateral salpingoopherectomy (TAH + BSO) and complete surgical staging was done. Histopathologic examination of Hematoxylin-eosin stained tissue samples revealed MMMT in right ovary (Figures 1 and 2) with metastasis to the left adnexa and omentum.

According to the International Federation of Gynecology and Obstetrics (FIGO) staging system (5), the patient was in stage IIIA. The serum CA-125 was elevated (386 IU/ $\mathrm{mL}$ ) but the level of CEA and CA19-9 were within normal limits (3.5 IU/mL and $9 \mathrm{IU} / \mathrm{mL}$, respectively).

The patient received six courses of chemotherapy consisting of carboplatin 6AUC and paclitaxel $\left(270 \mathrm{mg} / \mathrm{m}^{2}\right)$ regimen every three weeks. After six courses of chemotherapy, abdominopelvic computed tomography (CT) findings were normal and there was no evidence of residual tumor in the pelvic cavity. CA-125 reverted to normal levels after surgery and six courses of chemotherapy. The patient was followed up every three months by pelvic examination, abdominal and pelvic sonography, and CA-125 
Homaei Shandiz F et al.

tumor marker. After 31-month disease-free survival, a nodule of about $1.5-\mathrm{cm}$ diameter was palpated in presacral region on rectal and vaginal examination. In July 2010, a round, hypoechoic solid mass of $16 \times 18 \mathrm{~mm}$ at the posterior right part of the rectum was found in transvaginal ultrasonography. Magnetic resonance imaging confirmed the existence of a mass of 17-mm diameter, compatible with a lymph node. CA125 level was increased (65 IU/mL). We recommended transrectal ultrasound-guided biopsy but the patient did not accept. In May 2011, a CT scan showed persist lymphadenopathy of $21 \times 11 \mathrm{~mm}$ in the

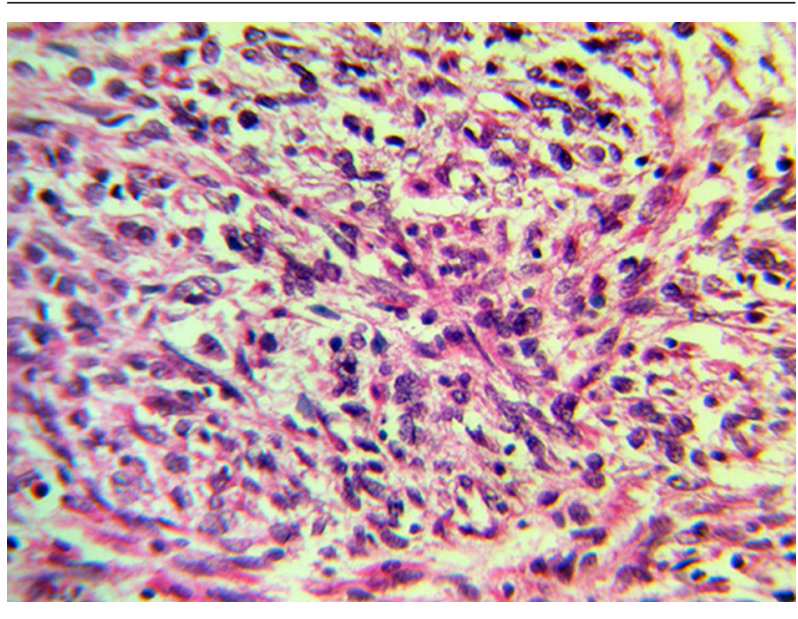

Figure 1. Histopathologic Examination of the Mass (Hematoxylin and Eosin Staining) presacral region. In June 2011 and after 42 months from the primary surgery, a CT-guided biopsy of the presacral mass was performed and histopathologic examination revealed a metastatic carcinoma. The patient underwent chemotherapy with primary regimen again (carboplatin 6 AUC and 270-mg/m² paclitaxel) every three weeks and 50.4 Gy radiotherapy in 28 fractions to a standard pelvic box field with photon of $15 \mathrm{mV}$. After 26 months followup, she became progression free with normal abdominopelvic CT findings and normal levels of tumor marker (CA-125) (Table 1).

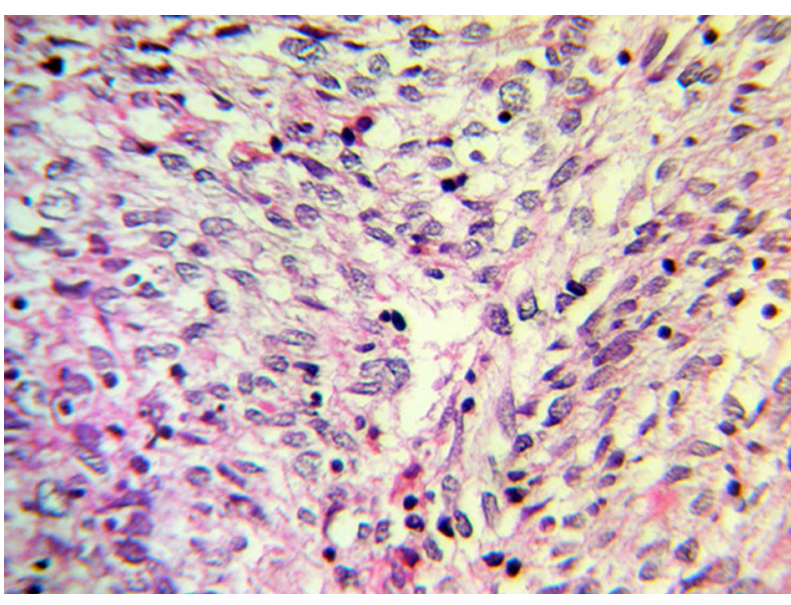

Figure 2. Histopathologic Examination of the Mass (Hematoxylin and Eosin Staining)

Table 1. History of Disease Progression and Treatments

\begin{tabular}{lc}
\hline Date & History \\
\hline June 2007 & Diagnosis of the disease (surgery + chemotherapy) \\
June 2008 & Free of disease \\
June 2010 & A mass of $16 \times 18 \mathrm{~mm}$ (tumor recurrence) was diagnosed \\
May 2011 & Persist lymphadenopathy of $21 \times 11 \mathrm{~mm}, \mathrm{CT}$-guided biopsy, metastatic carcinoma, 50.4 Gy radiotherapy \\
$\mathbf{2 6}$ Months Later & Free of disease \\
\hline
\end{tabular}

\section{Discussion}

MMMT is a rare and very aggressive cancer of female genital tract, seen mostly in the uterus and very rarely on the ovaries (ovarian carcinosarcoma, OCS) (6). On histopathologic examination, these tumors are characterized by a benign or atypical epithelial component and an invariably, low-grade, malignant stromal component (7). The median age at diagnosis of OCS is more than that of epithelial ovarian cancer (EOC) (8). Risk factors for MMMT include obesity, nulliparity, exogenous estrogen, and long-term tamoxifen use (3). OCS occurs in older women and more frequently in patients with disseminated disease. The survival for both early-stage and latestage carcinosarcoma is shorter than serous tumors (9).
We have presented a 64-year-old woman with none of the known risk factors who was diagnosed with stage III ovarian MMMT. We did optimal cytoreductive surgery and postoperation platinum-based chemotherapy. She was followed by ultrasonography, CA125, and physical examination. The pelvic recurrence was diagnosed 42 months later and was successfully treated by chemotherapy and radiotherapy. At the time of writing the report, she was tumor free for 24 months, which was a predominantly good survival for a metastatic case of MMT.

In the most recent reports, optimal cytoreductive surgery has been associated with improved outcome (8). Platinum-based chemotherapy is recommended follow- 
ing primary surgical debulking $(10,11)$. However, there is controversial about the effectiveness of adjuvant radiotherapy (12). Our patient was treated with the platinumbased chemotherapy as well. In a review article that was published in 2011, it was believed that the benefits of radiation therapy in the treatment of OCS lacks enough data and is mostly based on reports; in addition, the role of this treatment modality remains unknown and only useful in single, isolated pelvic recurrence (13). Callister et al. reported the results of 300 patients with MMMT treated with different modalities. The most common site of distant recurrence was the peritoneal cavity and they reported that adjuvant pelvic radiotherapy after surgery would decrease the risk of pelvic recurrence and might delay the appearance of distant metastasis (14). Mano et al. on 2007 reported that radiotherapy may be appropriate for patients with chemotherapy refractory, recurrent, or persisted disease restricted to the pelvic (15). In our patient, the pelvic recurrence was successfully treated by pelvic radiotherapy and she remained tumor free for 26 months. There is also controversy regarding the role and prognostic significance of tumor markers (CEA, CA125, CA14-4, CA15-3) (16). In a retrospective study, it was reported that tumor markers had no significant role (13). There are still different results in the literatures that consider CA125 as a useful marker for following therapy in ovarian MMMT (17). In our patient, CA125 was useful in follow-up and had significant rise after recurrence and decreased after successful treatment.

Carcinosarcoma contain both carcinomatous malignant epithelial and sarcomatous mesenchymal elements. Some studies have reported that the presence of heterologous sarcomatous elements is associated with a poor prognosis $(18,19)$. In our patient with relatively long survival tumor, the pathology report was highgrade MMM with homologous predominance of spindle cells sarcomatous component (fibrosarcoma) (Figure 2); the carcinomatous component was glandular (adenocarcinoma).

Ovarian MMMT is very rare and there is significant controversy over the treatment modalities, especially regarding the role of radiotherapy and the best time for its prescription. We have reported a patient with stage III OCS who was treated with complete surgery and platinum-based chemotherapy and then with chemoradiotherapy after recurrence. Fortunately, she had relatively good survival.

\section{Acknowledgements}

We wish to thank the staff of the Radiation Oncology Department of Mashhad University of Medical Sciences.

\section{Authors' Contributions}

1) Study concept and design: Sima Kadkhodayan. 2) Acquisition of data: Fatemeh Homaei Shandiz and Noorieh Sharifi Sistani. 3) Analysis and interpretation of data:
Sima Kadkhodayan. 4) Drafting of the manuscript: Zohre Yousefi. 5) Statistical analysis: Malihe Hsanzade Mofrad and Mahbobe Sadeghei. 6) Administrative, technical, and material support: Majid Nabizadeh Marvast. 7) Study supervision: Sima Kadkhodayan.

\section{Financial Disclosure}

We had no financial interests related to the material in the manuscript.

\section{Funding/Support}

The study was financially supported by Mashhad University of Medical Sciences.

\section{References}

1. Duman BB, Kara IO, Gunaldi M, Ercolak V. Malignant mixed Mullerian tumor of the ovary with two cases and review of the literature. Arch Gynecol Obstet. 2011;283(6):1363-8.

2. Arora P, Rao S, Khurana N, Talwar D, Tanwar R. Malignant mixed Mullerian tumor of broad ligament with synchronous ovarian and endometrial carcinoma: a rare association. J Cancer Res Ther. 2011;7(1):88-91.

3. Ma CJ, Yang SF, Huang CC, Chai CY, Cheng KI, Tsai EM, et al. Malignant mixed mullerian tumor of primary mesenteric origin associated with a synchronous ovarian cancer: case report and literature review. Eur J Gynaecol Oncol. 2008;29(3):289-93.

4. Nasser H, Morris RT, Fathallah L. Ovarian malignant mixed mullerian tumor with primitive neuroectodermal differentiation: case report with review of the literature. Pathol Res Pract. 2011;207(3):202-6.

5. FIGO. Announcement. Gyn Oncol J.1986;25(3):383-5.

6. Belbaraka R, Taleb A, Errihani H. A Rare Tumor of the Ovary: Carcinosarcoma. Med Case J. 2010;1(2):55-7.

7. Shah A, Rekhi B, Maheshwari A, Jambhekar NA. Low-grade Mullerian adenosarcoma with prominent decidualization, involving bilateral ovaries against a background of endometriosis: a diagnostic and treatment challenge.JPostgrad Med.2013;59(2):149-52.

8. del Carmen MG, Birrer M, Schorge JO. Carcinosarcoma of the ovary: a review of the literature. Gynecol Oncol. 2012;125(1):271-7.

9. George EM, Herzog TJ, Neugut AI, Lu YS, Burke WM, Lewin SN et al. Carcinosarcoma of the ovary: natural history, patterns of treatment, and outcome. Gynecol Oncol. 2013;131(1):42-5.

10. Rutledge TL, Gold MA, McMeekin DS, Huh WK, Powell MA, Lewin $\mathrm{SN}$, et al. Carcinosarcoma of the ovary-a case series. Gynecol Oncol. 2006;100(1):128-32.

11. Tate Thigpen J, Blessing JA, DeGeest K, Look KY, Homesley HD, Gynecologic Oncology G. Cisplatin as initial chemotherapy in ovarian carcinosarcomas: a Gynecologic Oncology Group study. Gynecol Oncol. 2004;93(2):336-9.

12. Kanthan R, Senger JL. Uterine carcinosarcomas (malignant mixed mullerian tumours): a review with special emphasis on the controversies in management. Obstet Gynecol Int. 2011;2011:470795.

13. Thomakos N, Rodolakis A, Zagouri F, Zacharakis D, Sotiropoulou M, Akrivos N, et al. Serum CA 125, CA 15-3, CEA, and CA 19-9: a prognostic factor for uterine carcinosarcomas? Arch Gynecol Obstet. 2013;287(1):97-102.

14. Callister M, Ramondetta LM, Jhingran A, Burke TW, Eifel PJ. Malignant mixed Mullerian tumors of the uterus: analysis of patterns of failure, prognostic factors, and treatment outcome. Int JRadiat Oncol Biol Phys. 2004;58(3):786-96.

15. Mano MS, Rosa DD, Azambuja E, Ismael G, Braga S, D'Hondt V, et al. Current management of ovarian carcinosarcoma. Int J Gynecol Cancer. 2007;17(2):316-24.

16. Menon S, Deodhar K, Rekhi B, Dhake R, Gupta S, Ghosh J, et al. Clin- 
ico-pathological spectrum of primary ovarian malignant mixed mullerian tumors (OMMMT) from a tertiary cancer institute: A series of 27 cases. Indian J Pathol Microbiol. 2013;56(4):365-71.

17. Peters WA, 3rd, Bagley CM, Smith MR. CA-125. Use as a tumor marker with mixed mesodermal tumors of the female genital tract. Cancer. 1986;58(12):2625-7.
18. Dictor M. Malignant mixed mesodermal tumor of the ovary: a report of 22 cases. Obstet Gynecol. 1985;65(5):720-4.

19. Sood AK, Sorosky JI, Gelder MS, Buller RE, Anderson B, Wilkinson EJ, et al. Primary ovarian sarcoma: analysis of prognostic variables and the role of surgical cytoreduction. Cancer. 1998;82(9):1731-7. 